\title{
Mothering matters: Maternal style predicts puppies' future performance
}

\author{
Lucia Lazarowski $^{1}$ - Jeffrey S. Katz ${ }^{1}$
}

Published online: 10 January 2018

(C) Psychonomic Society, Inc. 2018

\begin{abstract}
Bray et al. (2017, Proceedings of the National Academy of Sciences, 113(32), 9128-9133) recently showed that maternal interactions between service dog mothers and their puppies were predictive of puppies' future success as a candidate service dog. These findings prompt questions into the role of genetics and early experiences and may provide useful selection tools for working dog breeding programs.
\end{abstract}

Keywords Service dogs $\cdot$ Maternal style $\cdot$ Canine cognition

The influence of early life experiences on social development is an ongoing area of interest in both human and animal research. From classics like Harlow's studies on infant attachment in monkeys and Lorenz's observations of imprinting in baby chicks, we have known that early interactions between caregiver and offspring can have lasting psychological effects. The extent to which such experiences impact development, and which aspects are significant, remains questioned.

Also of interest is how cognitive abilities and temperament relate to, and possibly predict, future performance. The potential to predict behavior has obvious value in many settings, evidenced by the pervasive use of standardized testing, personality assessments, and personnel selection tools. The quest to choose the best candidate for a given role is not isolated to humans and is especially valued in the pet and working animal industry. Service dogs, for example, undergo rigorous training for several months before they are granted entry into advanced training for operational placement, and approximately one third of candidate dogs fail to do so. Given the costs and time associated with training, the ability to determine from an early age which dogs have a greater chance of succeeding is an ongoing challenge in the working dog industry.

To examine the effects of early experience and the role of cognition and temperament in the success of candidate service dogs, Bray, Sammel, Cheney, Serpell, \& Seyfarth (2017) studied the behavior of 21 mothers and their litters from a guide

Jeffrey S. Katz

katzjef@auburn.edu

1 Department of Psychology, Auburn University, 226 Thach Hall, Auburn, AL 36849, USA dog breeding and training program. Behavioral interactions between mothers and puppies during a 3-week postnatal period were observed. The puppies were then assessed upon returning to the training center as young adults after having spent more than a year in basic training and socialization in the homes of volunteer "puppy raisers" before formal training began. Assessments included 11 measures of cognitive performance and temperament, including attention, memory, problem solving, and reactions to novel objects, people, and situations. Maternal behavior and assessment measures were then correlated to final outcome (i.e., successful placement as a guide dog or failure due to behavioral reasons).

Maternal behavior for each mother was characterized by physical proximity and contact with her puppies as well as nursing position (i.e., lying down, sitting, or standing) during the 3-week postnatal period. High scores of maternal behavior (e.g., greater levels of interaction and proximity with puppies) were associated with poorer performance on the young adult cognitive and temperament assessments. These performance measures included restlessness when isolated, longer latencies to success and more errors on problem-solving tasks, and shorter latencies to vocalize when confronted with a novel object. Maternal behavior was also predictive of program outcome in that high levels of maternal care, and specifically ventral nursing styles (i.e., lying down on stomach), were predictive of failure from the program.

Young adult test performance was also predictive of program outcome. In particular, poor performance on a multistep problem-solving task, shorter latency to vocalize in response to a novel object, and reactivity to an umbrella opening were associated with release from the program. Interestingly, the direction of the umbrella test reaction depended on breed: 
Labrador retrievers with greater reactions to the umbrella opening were more likely to fail as a guide dog, whereas the opposite was true for Golden retrievers (i.e., greater reactions were associated with success). Overall, young adult test performance had greater predictive strength than did maternal behavior, though both were associated with program outcome.

In sum, the study found that increased levels of maternal care were associated with decreased cognitive performance, more anxious temperament, and greater likelihood of failure from the program. Placing these results within the framework of previous findings that stressful experiences early in life can have long-term effects on behavior, the authors suggested that moderate amounts of stress may actually be beneficial. In this case, reduced interactions between mother and puppies presents small challenges that the puppies must learn to overcome (e.g., increased effort to nurse from a sitting or standing position), which may better prepare the puppies later in life. The authors concluded that overbearing mothers do not allow the puppies sufficient exposure to moderate stressful experiences and associated opportunities to learn how to cope, which is reflected in their reduced cognitive abilities, anxious temperaments, and ultimately undesirable characteristics for a guide dog.

While it is possible that the dogs' cognitive and behavioral development was shaped by early experiences reflecting the degree of their mothers' involvement, other explanations are possible. As noted by the authors, a limitation of the study's design is the inability to control for possible confounding genetic effects. That is, the possibility that puppies' behavior was inherited from their mothers rather than determined by mothering style cannot be ruled out. A genetic predisposition to higher arousal may lead to a more anxious and overly attentive mothering style, with her puppies inheriting the same anxious temperament. In fact, the study found that mothers exhibiting high levels of maternal behavior also had increased cortisol levels and greater stress responses when separated from her litter. In order to control for possible genetic effects, the authors suggest cross-fostering studies in which puppies are raised by mothers other than their own. Another approach would be to experimentally manipulate mothering style by limiting or promoting interactions between mother and puppies, or by introducing structured challenges into the puppies' environment. The prediction here would be that if the effects of maternal style are due to the moderate stressful events accompanying the mothers' behavior, then other events inducing moderate stress should have similar effects on puppies' behavior. Consistent with this hypothesis, exposure to "mental challenges" and independent environmental socialization away from the litter during the first 6 weeks of life was recently shown to decrease future undesirable behaviors in service dog puppies (Vaterlaws-Whiteside \& Hartmann, 2017). On the other hand, although task specificity may come into play, Schoon and Berntsen (2011) found that introducing mild forms of stress through early neurological stimulation had no effect on development or performance in mine detection dogs. Clearly, more research into the effects of challenging experiences early in life is needed.

It is unclear how generalizable these findings are to other populations of working dogs. A previous study on the effect of maternal behavior in military working dogs (MWDs) found the opposite effect, in that increased maternal care was associated with more desirable outcomes in the puppies (Foyer, Wilsson, \& Jensen, 2016). These contradictory findings may align with the genetic account for effects of maternal behavior: MWDs are typically selected for traits associated with high motivational drives and arousal, whereas the opposite is true for service dogs, which tend to exhibit lower levels of baseline arousal. The traits associated with successful MWDs are likely to be reflected in overactive mothering styles but also produce offspring inheriting these traits.

Regardless of the mechanisms by which maternal style relates to cognition, temperament, and the future success of service dogs, characterizing maternal style appears to be a valuable tool for selecting breeders and candidate service dogs. Beyond such behavioral markers, biological markers will be an important addition to the selection process and may be useful in teasing apart genetic confounds. Gene mapping experiments will provide an excellent tool for not only understanding diseases and behavioral disorders but also for development and cognition. Additionally, awake neuroimaging is a relatively new and emerging method for exploring the predictive nature of neural functioning in dogs as it bears upon canine behavior, cognition, and working dog selection (Thompkins, Deshpande, Waggoner, \& Katz, 2016). Combining all these measures to discover biobehavioral markers will be important not only for selecting service dogs but also for understanding the evolution of cognitive function.

\section{Compliance with ethical standards}

Declarations of interest None

\section{References}

Bray, E. E., Sammel, M. D., Cheney, D. L., Serpell, J. A., \& Seyfarth, R. M. (2017). Effects of maternal investment, temperament, and cognition on guide dog success. Proceedings of the National Academy of Sciences, 113(32), 9128-9133.

Foyer, P., Wilsson, E., \& Jensen, P. (2016). Levels of maternal care in dogs affect adult offspring temperament. Scientific Reports, 6, 19253.

Schoon, A., \& Berntsen, T. G. (2011). Evaluating the effect of early neurological stimulation on the development and training of mine detection dogs. Journal of Veterinary Behavior: Clinical Applications and Research, 6(2), 150-157.

Thompkins, A. M., Deshpande, G., Waggoner, P., \& Katz, J. S. (2016). Functional magnetic resonance imaging of the domestic dog: Research, methodology, and conceptual issues. Comparative Cognition and Behavior Reviews, 11, 63-82.

Vaterlaws-Whiteside, H., \& Hartmann, A. (2017). Improving puppy behavior using a new standardized socialization program. Applied Animal Behaviour Science, 197, 55-61. 